\title{
CircWAC induces chemotherapeutic resistance in triple-negative breast cancer by targeting miR-142, upregulating WWP1 and activating the PI3K/AKT pathway
}

Lei Wang ${ }^{1+}$, Yehui Zhou ${ }^{1+}$, Liang Jiang ${ }^{2}$, Linlin Lu' ${ }^{1}$, Tiantian Dai ${ }^{1}$, Aoshuang Li ${ }^{1}$, Yan Chen ${ }^{1 *}$ (D) and Lifeng Zhang ${ }^{1 *}$

\begin{abstract}
Background: Chemotherapeutic resistance is the main cause of clinical treatment failure and poor prognosis in triple-negative breast cancer (TNBC). There is no research on chemotherapeutic resistance in TNBC from the perspective of circular RNAs (circRNAs).

Methods: TNBC-related circRNAs were identified based on the GSE101124 dataset. Quantitative reverse transcription PCR was used to detect the expression level of circWAC in TNBC cells and tissues. Then, in vitro and in vivo functional experiments were performed to evaluate the effects of circWAC in TNBC.

Results: CircWAC was highly expressed in TNBC and was associated with worse TNBC patient prognosis. Subsequently, it was verified that downregulation of circWAC can increase the sensitivity of TNBC cells to paclitaxel (PTX) in vitro and in vivo. The expression of miR-142 was negatively correlated with circWAC in TNBC. The interaction between circWAC and miR-142 in TNBC cells was confirmed by RNA immunoprecipitation assays, luciferase reporter assays, pulldown assays, and fluorescence in situ hybridization. Mechanistically, circWAC acted as a miR-142 sponge to relieve the repressive effect of miR-142 on its target WWP1. In addition, the overall survival of TNBC patients with high expression of miR-142 was significantly better than that of patients with low expression of miR-142, and these results were verified in public databases. MiR-142 regulated the expression of WWP1 and the activity of the PI3K/AKT pathway. It was confirmed that WWP1 is highly expressed in TNBC and that the prognosis of patients with high WWP1 expression is poor.
\end{abstract}

Conclusions: CircWAC/miR-142/MWP1 form a competing endogenous RNA (ceRNA) network to regulate PI3K/AKT signaling activity in TNBC cells and affect the chemosensitivity of cells.

Keywords: circWAC, miR-142, WWP1, PI3K/AKT, TNBC

\footnotetext{
*Correspondence: walves@suda.edu.cn; trainhead008@163.com

${ }^{+}$Lei Wang and Yehui Zhou contributed equally to this work.

'Department of General Surgery, The First Affiliated Hospital of Soochow University, 188 Shizi Street, Suzhou 215000, China

Full list of author information is available at the end of the article
}

(c) The Author(s). 2021 Open Access This article is licensed under a Creative Commons Attribution 4.0 International License, which permits use, sharing, adaptation, distribution and reproduction in any medium or format, as long as you give appropriate credit to the original author(s) and the source, provide a link to the Creative Commons licence, and indicate if changes were made. The images or other third party material in this article are included in the article's Creative Commons licence, unless indicated otherwise in a credit line to the material. If material is not included in the article's Creative Commons licence and your intended use is not permitted by statutory regulation or exceeds the permitted use, you will need to obtain permission directly from the copyright holder. To view a copy of this licence, visit http://creativecommons.org/licenses/by/4.0/ The Creative Commons Public Domain Dedication waiver (http://creativecommons.org/publicdomain/zero/1.0/) applies to the data made available in this article, unless otherwise stated in a credit line to the data. 


\section{Background}

As a malignancy arising in breast epithelial tissue, approximately $99 \%$ of breast cancers occurs in women. According to GLOBOCAN data, in 2018, there were approximately 2.1 million new cases of breast cancer worldwide, accounting for $11.6 \%$ of the total number of cancer diagnoses and approximately 630,000 deaths, accounting for $6.6 \%$ of the total number of cancer deaths; thus, breast cancer had the highest incidence among female cancers [1]. The incidence and mortality rates of breast cancer in China increased annually from 2003 to 2014, seriously affecting women's health and life expectancy [2, 3]. In China, there were approximately 270,000 new cases of breast cancer and 70,000 deaths in 2015 [4]. Triple-negative breast cancer (TNBC), accounting for $15-20 \%$ of breast cancers, is one of the most unique types of breast cancer, and its biological behavior, onset characteristics and prognosis are different from those of other molecular subtypes of breast cancer [5]. TNBC tissues are negative for ER, PR and HER-2; thus, their sensitivity to endocrine therapies, molecular targeted therapies and chemotherapeutics is reduced, increasing the difficulty of clinical treatment. To date, surgery, radiotherapy and chemotherapy are the main treatments for TNBC. In clinical practice, adjuvant chemotherapy, such as anthracycline and paclitaxel (PTX)-based chemotherapy regimens, is still the main treatment method, but the development of chemotherapeutic resistance is still the main cause of clinical treatment failure and poor prognosis in breast cancer patients, and the 5-year survival rate of TNBC is significantly lower than that of non-TNBC breast cancers [6]. Therefore, it is extremely urgent to improve the efficacy of TNBC therapies and prolong the patient survival time.

Currently, with the development of high-throughput sequencing techniques, RNomics has gradually become a focus of attention. Circular RNAs (circRNAs) are a new type of RNA molecule that differ from traditional linear RNAs. They have a closed loop structure and are abundant in eukaryotic transcriptomes. CircRNAs are usually formed by cleavage and circularization of host gene exons or introns. Their closed loop structure is not sensitive to RNase R; thus, they are more stable than linear RNAs and can be used as tumor markers and potential targets in clinical applications [7]. In recent studies, it has been found that circRNAs play an important regulatory role in tumorigenesis and tumor development. For example, ciRS-7 upregulates the expression of oncogenes such as mTOR, EGFR and PIK3CD by sponging miR-7 and participates in the progression of neuroblastoma and renal cancer [8]. Research on breast cancer-related circRNAs is in its infancy [9], and circRNAs related to chemosensitivity in TNBC have not been reported. Therefore, further exploration of chemotherapeutic resistance in TNBC from the perspective of circRNAs will help reveal novel mechanisms underlying the occurrence and development of PTX resistance in TNBC, and it can provide new ideas for clinical diagnosis and treatment.

In the present study, the differentially expressed circRNAs in TNBC were screened, and TNBC-related circRNAs were identified based on the GSE101124 dataset [10]. Combining these results with the results in TNBC patient specimens from the First Affiliated Hospital of Soochow University, it was found that circWAC was highly expressed in TNBC and that its expression level was positively correlated with the prognosis of TNBC; that is, the higher the expression of circWAC, the worse was the prognosis of TNBC patients. In vivo and in vitro experiments confirmed that downregulation of circWAC in TNBC increased the sensitivity of cells to PTX chemotherapy. Subsequently, mechanistically, it was confirmed that circWAC indirectly upregulated the expression of WWP1, the target gene of miR-142, and activated the PI3K/AKT pathway by competitively binding to miR-142, thus leading to chemotherapeutic resistance in TNBC.

\section{Methods}

\section{TNBC tissue samples}

The tissue samples used in this study were obtained from TNBC patients undergoing surgical treatment at the First Affiliated Hospital of Soochow University. The surgical specimens were cryopreserved in liquid nitrogen after isolation. Thirty paired TNBC tumor/paracancer tissue samples were randomly selected to detect the differential expression of circRNAs (none of the patients received any other treatments, including radiotherapy, chemotherapy or targeted drug therapy, prior to surgery). In addition, 90 TNBC patients who underwent radical mastectomy between 2013 and 2015 at the First Affiliated Hospital of Soochow University and received at least 4 cycles of PTX adjuvant chemotherapy were selected. Postoperative pathological diagnostic reporting was performed by two pathologists from the First Affiliated Hospital of Soochow University. The study patients and their families were informed of the research during the preoperative conversation, and a doctor-patient informed consent form was signed. The research project complied with the Declaration of Helsinki and was approved by the Ethics Committee of the First Affiliated Hospital of Soochow University.

\section{Cell lines}

The cell lines MDA-MB-231, MDA-MB-468, HCC1937, MDA-MB-361, MCF-7 and MCF-10A used in this 
experiment were purchased from ATCC. Cells were cultured in an incubator at $37^{\circ} \mathrm{C}$ with $5 \% \mathrm{CO}_{2}$ and saturated humidity.

\section{Quantitative reverse transcription-polymerase chain reaction (qRT-PCR)}

TRIzol reagent was used to extract total RNA from tissues and cells, and a NanoDrop 2000 microspectrophotometer was used to determine the quality and concentration of total RNA. A PrimeScript RT Reagent Kit was used for reverse transcription to synthesize cDNA. Fluorescence quantitative PCR was performed using TB Green Fast qPCR Mix. All primers were designed and synthesized by Tingzhou Bio (Shanghai, China). Related primer sequences are shown in Supplementary Table 1.

\section{Western blot analysis}

Total cellular protein was extracted, and protein quantification was performed with a protein quantification kit. After quantification, $20 \mu \mathrm{g}$ of the samples was loaded on a $12 \%$ SDS-PAGE gel, separated, transferred to a PVDF membrane, blocked with skim milk $(50 \mathrm{~g} / \mathrm{L})$, washed, and incubated with primary antibodies (1:500) and secondary antibodies (1:2000), and immunoreactions were then visualized with ECL reagents. With the GAPDH and $\beta$-actin proteins as internal references, the optical density (OD) values of relevant proteins (WWP1, PTEN, $\mathrm{AKT}, \mathrm{pS} 473-\mathrm{AKT}$, etc.) were analyzed using Quantity One software.

\section{Cell transfection}

The circWAC expression plasmid, circWAC small interfering RNA (siRNA), WWP1 siRNA, control constructs, negative control miRNA (miR-NC), miR-142 mimic, and miR-142 inhibitor were purchased from XimaoBio (Shanghai, China). According to the instructions, cells were transfected using Lipofectamine 3000.

\section{Dual luciferase reporter assay}

To experimentally predict the possible miRNA target genes and binding sequences, the wild-type and mutant sequences were synthesized and cloned into dual luciferase reporter plasmids (Promega) containing the psiCheck2 promoter. After MDA-MB-231 cells were inoculated into a 96-well plate and cultured for $24 \mathrm{~h}$, they were cotransfected with the wild-type or mutant reporter gene plasmids and overexpression or silencing plasmid mimics. Luciferase activity was measured $48 \mathrm{~h}$ after transfection.

\section{CCK-8 assay}

Cells in logarithmic growth phase were fully and evenly spread in a 96-well plate and cultured for $24 \mathrm{~h}$. After $24 \mathrm{~h}$ of drug treatment and/or transfection, the medium was replaced, and CCK- 8 reagent at a final concentration of $10 \%$ was then added to the cultures. After $1-4 \mathrm{~h}$, the absorbance at $450 \mathrm{~nm}$ was measured using a microplate reader. Cell survival curves were plotted with the cell survival rate as the ordinate and the PTX concentration as the abscissa.

\section{Colony formation assay}

Cells were inoculated into a 6-well plate, the medium was replaced after $24 \mathrm{~h}$ of drug treatment and/or transfection, and the cells were then fixed with methanol for 15 min. Then, the fixative was removed, and Giemsa staining was performed for $20 \mathrm{~min}$.

\section{Apoptosis assay}

A FITC Annexin V Apoptosis Assay Kit was used for apoptosis detection as follows. Cells in logarithmic growth phase were collected, resuspended and evenly spread in a 6-well plate, and cultured for $24 \mathrm{~h}$. After $24 \mathrm{~h}$ of transfection and/or drug treatment, the supernatant was removed, and the cells were then washed twice with PBS, trypsinized and resuspended in HEPES buffer (containing calcium). Then, the tumor cells were stained with Annexin V-FITC and PI for $15 \mathrm{~min}$ in the dark. After washing, apoptosis was detected by flow cytometry.

\section{Cell invasion and metastasis assays}

After $24 \mathrm{~h}$ of transfection treatment, the cells were prepared into a cell suspension $\left(1 \times 10^{5} / \mathrm{mL}\right)$ with culture medium. One hundred microliters of the suspension was collected and inoculated into the upper Transwell chamber, and $500 \mu \mathrm{L}$ of medium containing $10 \%$ fetal bovine serum was added into the lower Transwell chamber. After $24 \mathrm{~h}$ of incubation at $37^{\circ} \mathrm{C}$, the cells in the upper chamber were wiped off with cotton swabs; the cells in the lower chamber were fixed with $4 \%$ paraformaldehyde, and their nuclei were stained with DAPI. Five fields were randomly selected under a microscope (100x magnification) to calculate the average number of migrated cells. In the cell invasion assay, the membrane in the upper Transwell chamber was coated with prediluted Matrigel, and the plate was incubated at room temperature for $6 \mathrm{~h}$. The remaining steps were the same as those in the cell migration assay.

\section{Fluorescence in situ hybridization (FISH)}

FISH was performed on MDA-MB-231 cells to determine the subcellular location of circWAC and miR-142. The cell specimens were placed in PBS containing 10\% fixative solution for 5 min for predenaturation. The slide specimens were immersed in the fixative solution twice for $10 \mathrm{~min}$ each, and the specimens were then incubated 
in 70, 90 and $100 \%$ ice-cold ethanol and dried. Fifty milliliters of $50 \%$ formamide $/ 2 \times \mathrm{SSC}$ was poured into a humidified chamber and preheated at $37^{\circ} \mathrm{C}$. The FITClabeled circWAC probe and PE-labeled miR-142 probe (Tingzhou, Shanghai, China) were detected and observed by confocal microscopy. The probe sequences are shown in Supplementary Table 2.

\section{Nucleocytoplasmic fractionation}

MDA-MB-231 cells were seeded in a $10 \mathrm{~cm} \times 10 \mathrm{~cm}$ culture dish, and the cells were collected at $90 \%$ confluence. A PARIS kit was used to separate the nuclear and cytoplasmic components, and the cells were trypsinized after washing with PBS. The cells were transferred into a 2 $\mathrm{mL}$ EP tube and centrifuged at $500 \mathrm{r} / \mathrm{min}$ and $4{ }^{\circ} \mathrm{C}$ for 5 $\mathrm{min}$, and the supernatant was then discarded. Then, $500 \mu \mathrm{L}$ of fractionation buffer was added and gently mixed by pipetting, and the samples were placed on ice for $5 \mathrm{~min}$ and then centrifuged again under the same conditions. The supernatant was the cytoplasmic fraction, and the precipitate was the nuclear fraction. RNA extraction and quantitative analysis were performed on the nuclear and cytoplasmic fractions. U6 was used as the internal reference for the nuclear fraction, and GAPDH was used for the cytoplasmic fraction.

\section{RNA immunoprecipitation (RIP)}

RNA enrichment was evaluated by qRT-PCR using a Magna RIP RNA binding protein immunoprecipitation kit (Millipore, Billerica, MA, USA) according to the manufacturer's instructions with an anti-Ago2 antibody or IgG as the control.

\section{Immunohistochemical staining (IHC)}

Paraffin tissue blocks were cut into $4 \mu \mathrm{m}$ slices and mounted on a glass slide. The slices were dewaxed in xylene and hydrated through a graded series of ethanols. Then, the slices were immersed in a citric acid antigen retrieval solution and incubated in a microwave oven for $15 \mathrm{~min}$ for antigen retrieval. The samples were incubated with a $3 \%(\mathrm{v} / \mathrm{v}) \mathrm{H}_{2} \mathrm{O}_{2}$ at $37^{\circ} \mathrm{C}$ for $15 \mathrm{~min}$ to inhibit endogenous peroxidase activity, after which they were blocked using 10\% goat serum and incubated at room temperature for $30 \mathrm{~min}$. Then, the slices were incubated with a rabbit anti-human WWP1 monoclonal antibody overnight at $4{ }^{\circ} \mathrm{C}$. The negative control slice was treated with phosphate-buffered saline (PBS) for $2 \mathrm{~h}$ under the same conditions. Then, the samples were incubated with the secondary antibody for $1 \mathrm{~h}$ prior to color development (ZSGB-Bio) and counterstaining with hematoxylin for $2 \mathrm{~min}$. The results were assessed by the semiquantitative integration method. Five high-power fields were randomly selected in the area highly populated with cells, and 100 cells were counted. The percentage of positively stained cells was scored as follows: $<5 \%$ positively stained cells, 0 points; $5-25 \%, 1$ point; $26-50 \%, 2$ points; $51-75 \%, 3$ points; and more than $75 \%$, 4 points. The staining intensity score was assigned as follows: no staining, 0 points; light brownish-yellow staining, 1 point; brownish-yellow staining, 2 points; and brown staining, 3 points. The immunohistochemical score was calculated as the product of the positive percentage score and the staining intensity score.

\section{Animal experiments}

Lentiviral vectors were used to generate MDA-MB-231/ sh-NC cells and MDA-MB-231/sh-circWAC cells, which had downregulated circWAC expression. sh-NC $(0.2 \mathrm{~mL}$, $\left.1 \times 10^{7}\right)$ and sh-circWAC $\left(1 \times 10^{7}\right)$ cells were injected into the backs of BALB/c nude mice to generate 10 mice bearing implanted tumors. The size of the implanted tumors was observed every $3 \mathrm{~d}$, and the volume of the tumors was calculated as $(\mathrm{V})=\left(\mathrm{LxW}^{2}\right) / 2$. On $\mathrm{d} 21$, the mice were randomly divided into 4 groups: sh-NC + control, sh-circWAC + control, MDA-MB-231/sh-NC + PTX, and sh-circWAC + PTX. Mice in the PTX group received intravenous injection of PTX $(20 \mathrm{mg} / \mathrm{kg})$ every $3 \mathrm{~d}$ for 5 cycles. After $18 \mathrm{~d}$ of treatment, all nude mice were sacrificed, and the tumor tissues were removed for evaluation of indexes.

\section{Statistical methods}

Statistical analysis was performed using GraphPad Prism 6.0. The experimental data are expressed as the mean \pm standard error of the mean (mean \pm SEM) values. The $t$ test was used to evaluate differences between two groups, and one-way analysis of variance (ANOVA) was used to evaluate differences among multiple groups. $p<0.05$ was considered to be statistically significant, and each experiment was repeated at least 3 times.

\section{Results \\ Differential expression of circRNAs in TNBC}

To study the differentially expressed circRNAs in TNBC tumor tissues and normal breast tissues, the GSE101124 dataset from the GEO database was used [10]. GSE101124 contains high-throughput array data of circRNAs in 4 TNBC tumor tissues and 3 normal breast tissues (Agilent-069978 Arraystar Human CircRNA microarray V1). With a fold change $>3.0$ and a t test $p<0.05$ as the threshold criteria, 8 upregulated circRNAs (hsa_circ_0000516, hsa_circ_0000517, hsa_circ_0000520, hsa_circ_0000519, hsa_circ 0007503, hsa_circ_0008784, hsa_circ_0005699 and hsa_circ_0004780) and 11 downregulated circRNAs (hsa_circ_0000376, hsa_circ_0001455, hsa_circ 0020080, hsa_circ_0004781, hsa_circ_0043278, hsa_ 
circ_0006220, hsa_circ_0000977, hsa_circ_0008911, hsa_circ_0005265, hsa_circ_0065173 and hsa_circ_ 0008303) were identified (Fig. 1a). From the above circRNAs, we selected the 5 most upregulated circRNAs (hsa_circ_0000517, hsa_circ_0000520, hsa circ_0008784, hsa_circ_0007503, hsa_circ_0000519) and the 5 most downregulated circRNAs (hsa_circ 0043278, hsa_circ_0006220, hsa_circ_0000977, hsa circ_0065173 and hsa_circ_0008911) for further research. Thirty samples of TNBC tumor tissues and adjacent tissues were randomly selected from the biological sample bank of the First Affiliated Hospital of Soochow University for qRT-PCR analysis. The results showed that the expression of hsa_circ_0007503 in TNBC tumor tissues was significantly higher than that in adjacent tissues. This circRNA was upregulated 11.33-fold and had the highest uniformity and the most significantly different expression (Fig. 1b). As a result, hsa_circ_0007503 in TNBC was initially selected as the research object, and its clinical significance was evaluated. Hsa_circ_0007503 originates from the WAC gene and is hereafter referred to as circWAC.

To study the clinical significance of the expression of circWAC in TNBC, qRT-PCR was used to detect the correlation between the expression level of circWAC in 90 samples of TNBC and the clinicopathological characteristics of the corresponding patients. The cutoff criterion for high and low expression of the circRNA was the median value. The results of statistical analysis showed that circWAC was not significantly related to patient age, tumor differentiation, tumor size, or lymph node metastasis. However, the expression level of circWAC was significantly related to the prognosis of the TNBC patients. The higher the expression of circWAC, the worse was the overall survival (OS) of patients (Fig. 1c).

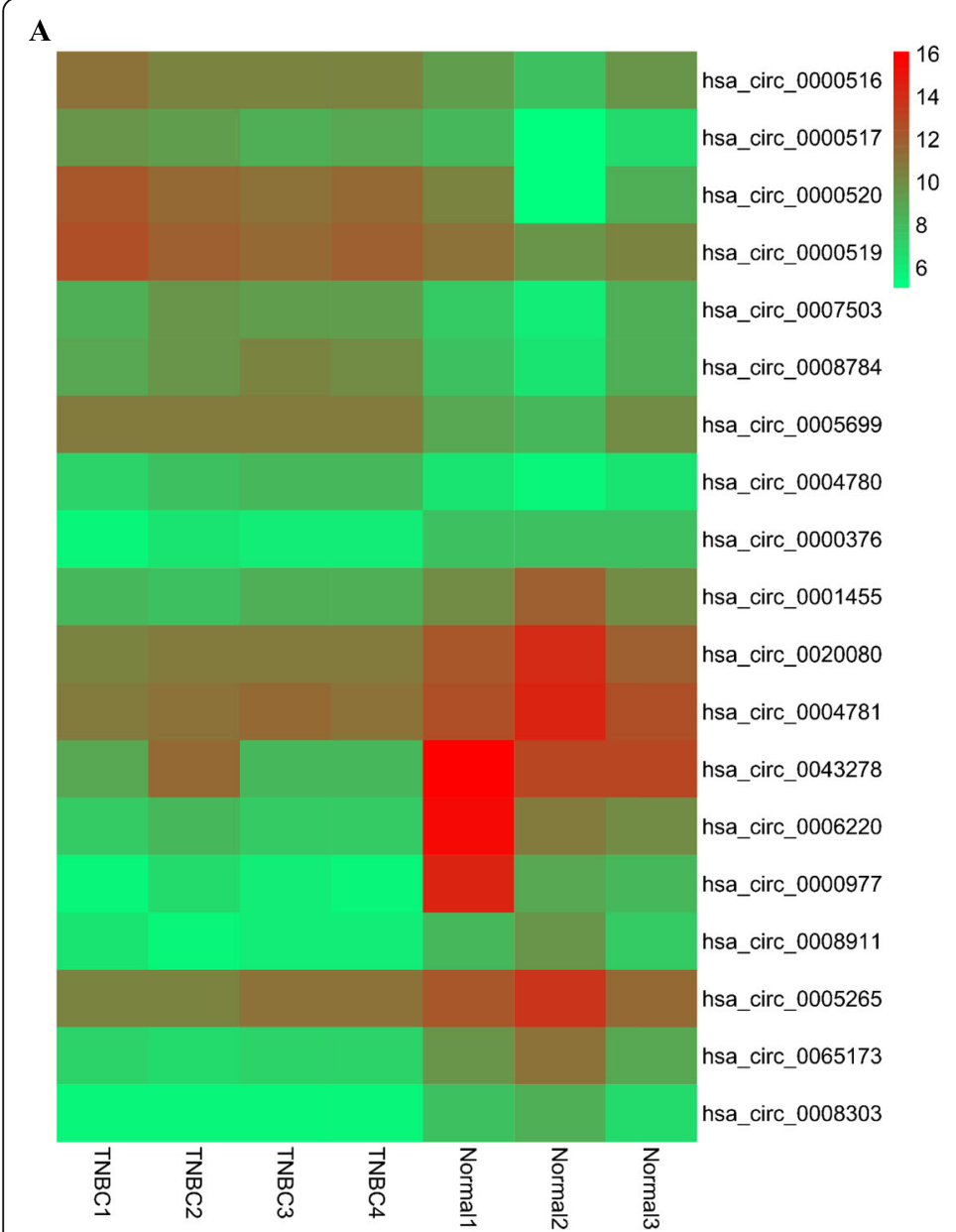

B

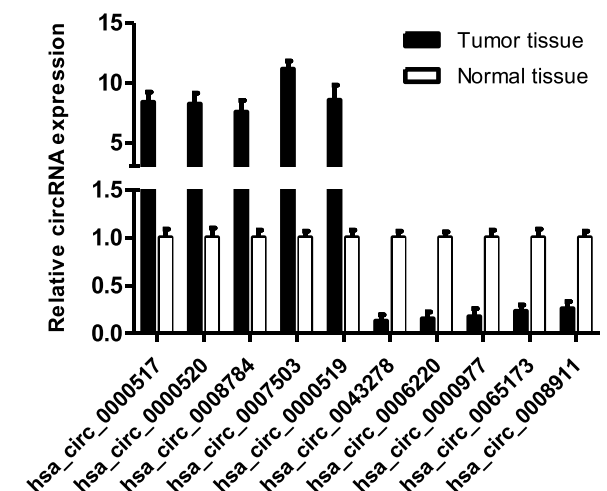

C

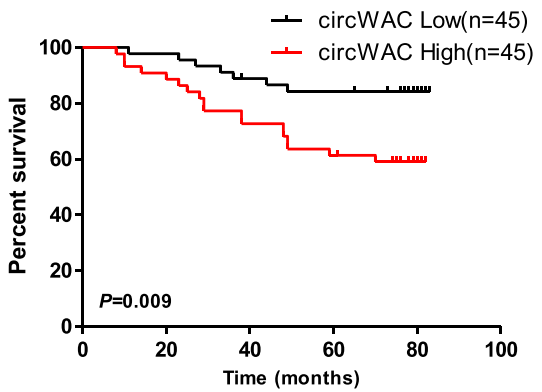

Fig. 1 The expression and prognostic significance of circWAC in TNBC. a The heat map shows the 19 most upregulated and downregulated circRNAs in TNBC tissues compared with normal breast tissues in the GSE101124 dataset. $\mathbf{b}$ The top five upregulated circRNAs and top five downregulated circRNAs in TNBC tissues were verified by qRT-PCR. $\mathbf{c}$ Kaplan-Meier survival curves indicating the correlation between circWAC and overall survival in TNBC. ${ }^{*} P<0.05,{ }^{* *} P<0.01,{ }^{* * *} P<0.001$ 


\section{Features of circWAC}

Databases such as circBase (http://www.circbase.org/) and the UCSC Genome Browser (http://genome.ucsc. $\mathrm{edu} /$ ) showed that circWAC is formed by the circularization of exons 4-7 of the WAC gene, that the spliced length is $645 \mathrm{bp}$ and that it is an exonderived circRNA. The results of Sanger sequencing confirmed head-to-tail splicing in the qRT-PCR product of circWAC (Fig. 2a). To prove that circWAC is circular rather than linear, we designed divergent and convergent primers for circWAC and the corresponding linear mRNA, respectively. Then, $\mathrm{cDNA}$ and gDNA were assayed by nucleic acid electrophoresis, and the results showed that circWAC is circular rather than linear (Fig. 2b). In addition, to confirm the stability of circWAC, we used RNase R treatment. Resistance to RNase R confirmed that circWAC has a circular structure (Fig. 2c). The results of actinomycin $\mathrm{D}$ treatment showed that the half-life of circWAC exceeded $24 \mathrm{~h}$, whereas that of the associated linear transcript was approximately $4 \mathrm{~h}$ (Fig. 2d). To determine the intracellular distribution of circWAC, we performed a nucleocytoplasmic fractionation experiment and found that circWAC is mainly distributed in the cytoplasm (Fig. 2e). Fluorescence in situ hybridization (FISH) showed the same result (Fig. 2f). The expression of circWAC was evaluated in a normal breast cell line (MCF-10A) and breast cancer cell lines (MDA-MB-231, MDA-MB-468, HCC-1937, MDA-MB361, MCF-7). The results showed that circWAC was

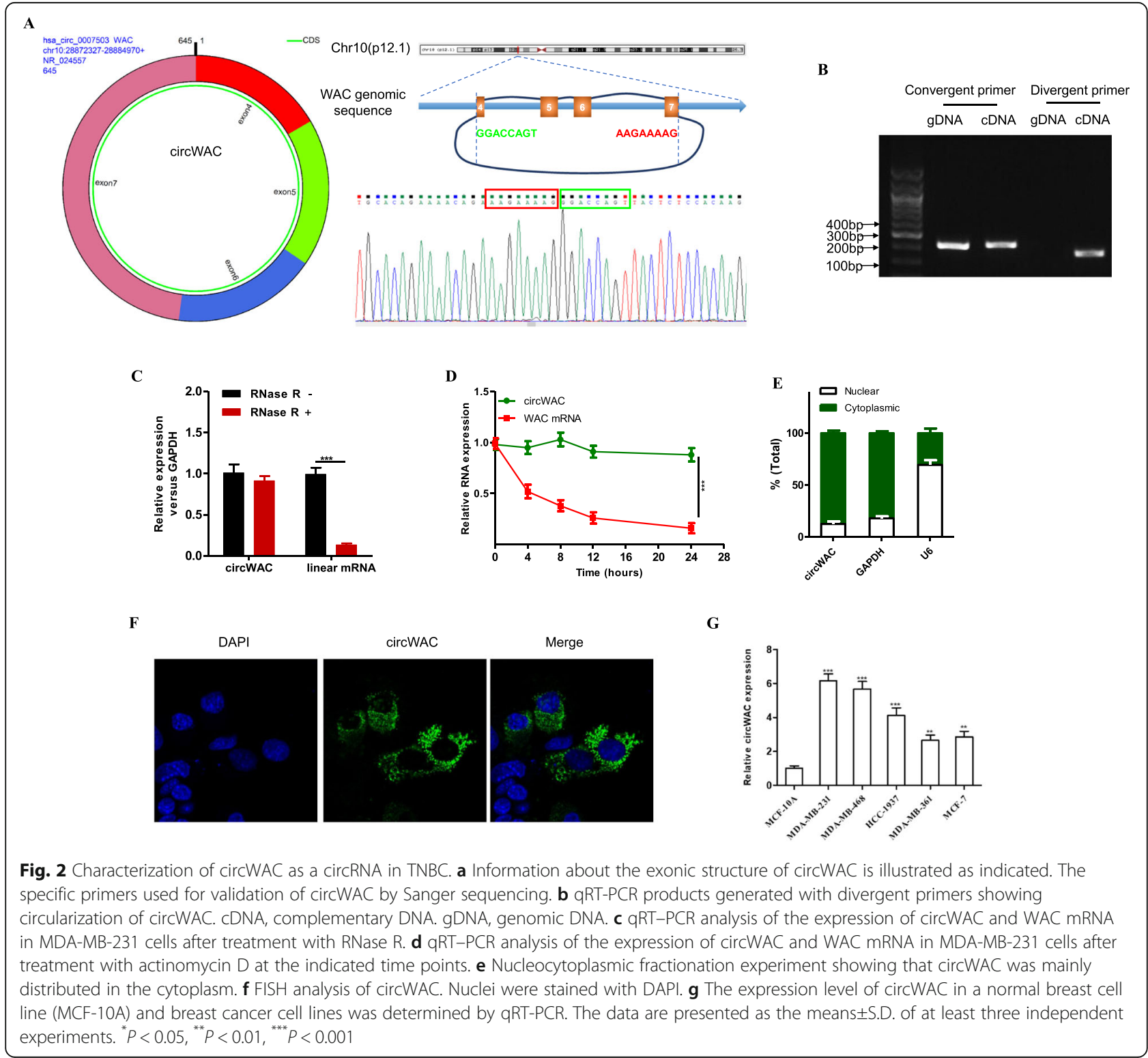


highly expressed in breast cancer cell lines and was most highly expressed in TNBC cell lines (MDA-MB-231, MDA-MB-468, HCC-1937) (Fig. 2g). The two TNBC cell lines with the highest expression levels of circWAC (MDA-MB-231 and MDA-MB-468) were selected for subsequent cell experiments.

\section{Function of circWAC in TNBC}

To study the function of circWAC in TNBC, the expression of circWAC was downregulated in the MDA-MB231 cell line, and the changes in cell functions were observed. The experimental data showed that circWAC had no obvious relationship with cell metastasis and invasion (Supplementary figure 1) but was closely related to the chemosensitivity of cells. Then, the role of circWAC in chemosensitivity was investigated in MDAMB-231 and MDA-MB-468 cells. MDA-MB-231 and MDA-MB-468 cells were transfected with a circWAC overexpression plasmid or siRNA. The expression levels of circRNA in cells after transfection are shown in Fig. 3a, b. The transfected cells were treated with different concentrations of PTX $(0,5,10,15,20,25,30 \mu \mathrm{mol} /$ L) for $24 \mathrm{~h}$, and a CCK- 8 assay was used to evaluate cell survival. Upregulation of circWAC decreased the sensitivity of cells to PTX (Fig. 3c, d), while downregulation of circWAC increased the sensitivity of cells to PTX (Fig. 3e, f). The IC50 values of PTX in MDA-MB-231 and MDA-MB-468 cells were $16.56 \mu \mathrm{mol} / \mathrm{L}$ and $20.31 \mu \mathrm{mol} / \mathrm{L}$, respectively. After overexpression of circWAC, the IC50 values of PTX in MDA-MB-231 and MDA-MB-468 cells increased to $23.43 \mu \mathrm{mol} / \mathrm{L}$ and $29.51 \mu \mathrm{mol} / \mathrm{L}$, respectively. After downregulation of circWAC, the IC50 values of PTX in MDA-MB-231 and MDA-MB-468 decreased to $11.42 \mu \mathrm{mol} / \mathrm{L}$ and $12.43 \mu \mathrm{mol} / \mathrm{L}$, respectively. Subsequent colony formation assays and apoptosis assays also confirmed that upregulation of circWAC reduced the sensitivity of cells to PTX, while downregulation of circWAC increased the sensitivity of cells to PTX (Fig. 3g-l). We also confirmed that circWAC could indeed increase the resistance of tumor cells to cisplatin (Supplementary Figure 2). In addition, we used 20 specimens from patients with metastatic TNBC in our specimen library. Ten patients were evaluated as $\mathrm{CR}$ or PR with target lesion reduction greater than $50 \%$ after 4 cycles of PTX-based chemotherapy, and they were deemed sensitive to PTX chemotherapy. The other 10 patients with tumor progression or reduction of less than $30 \%$ after 4 cycles of PTX-based

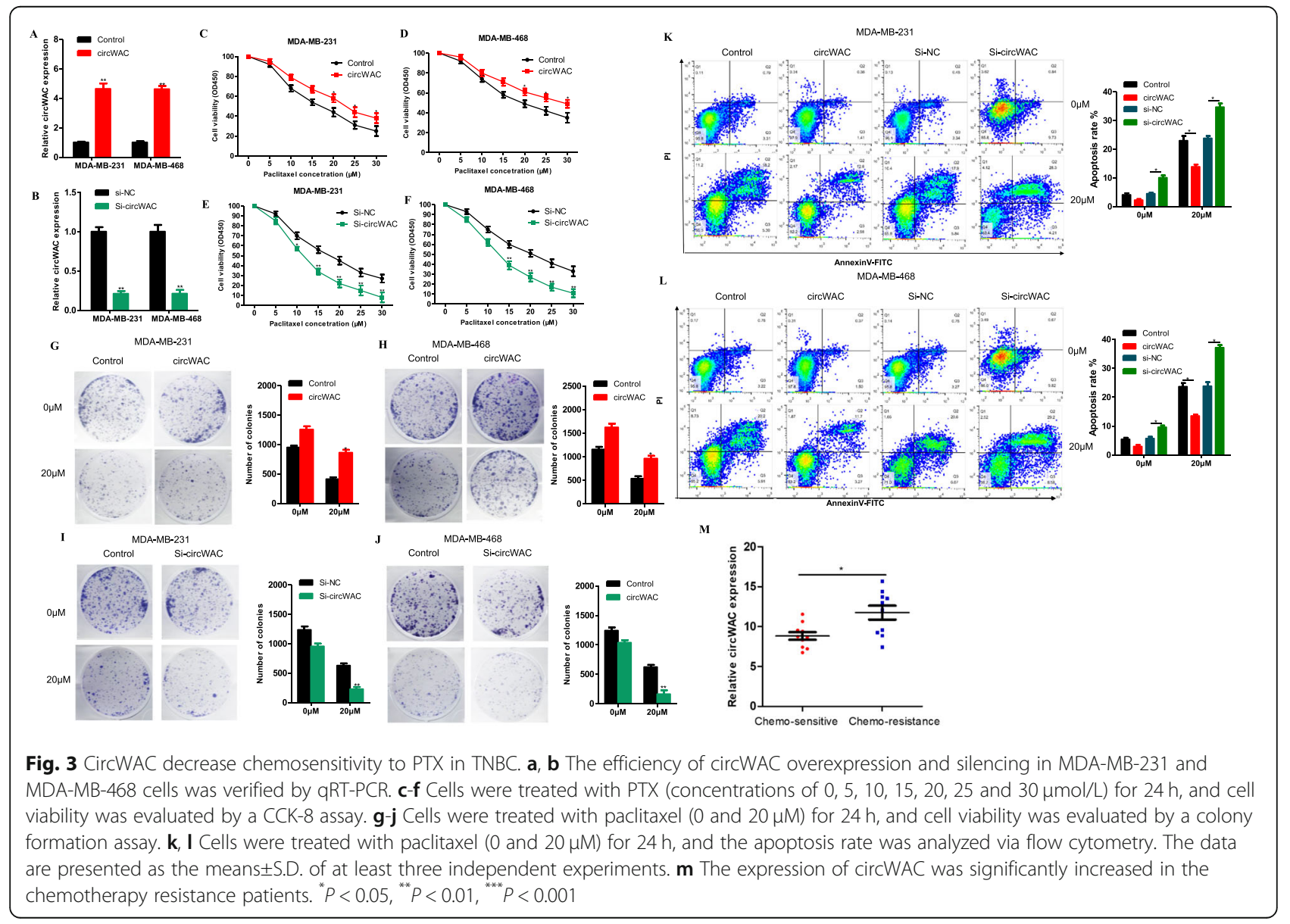


chemotherapy were regarded as chemotherapy resistance. We found that the expression of circWAC was significantly increased in the chemo-resistance group (Fig. 3m).

\section{CircWAC regulates PTX resistance by targeting miR-142}

The competing endogenous RNA (ceRNA) mechanism, which mainly regulates gene expression at the posttranscriptional level, is currently the most widely reported circRNA regulatory mechanism. The online analysis software associated with the Circular RNA Interactome (https://circinteractome.nia.nih.gov/) and circBANK (http://www.circbank.cn/) databases was used for bioinformatic analysis to screen miRNAs according to their RNA binding scores. It was found that miR-593, miR383, miR-498, miR-142-5p (hereafter referred to as miR142 ) and miR-1183 are miRNAs potentially sponged by circWAC. Subsequently, the expression level of circWAC in MDA-MB-231 and MDA-MB-468 cells was upregulated or downregulated, and among the above identified miRNAs, only the expression level of miR-142 changed (Fig. 4a, b), suggesting that miR-142 is a miRNA potentially sponged by circWAC. To observe the colocalization of circWAC and miR-142 in cells, FISH was performed. The results showed that circWAC and miR-142 colocalized in MDA-MB-231 cells mainly in the cytoplasm (Fig. 4c). RNA immunoprecipitation was performed to observe whether circWAC can bind to miR-142. The RIP results showed higher levels of circWAC and miR-142 in the Ago2 antibody group than in the IgG control group (Fig. 4d), proving that circWAC can bind to miR-142. Then, plasmids containing the wild-type sequence (circWAC-WT) and the mutant binding site sequence (circWAC-MUT) were constructed (Fig. 4e) and cotransfected with the miR142 mimic or miR-NC into MDA-MB-231 cells for a dual luciferase reporter assay. The results showed that overexpression of miR-142 significantly reduced the

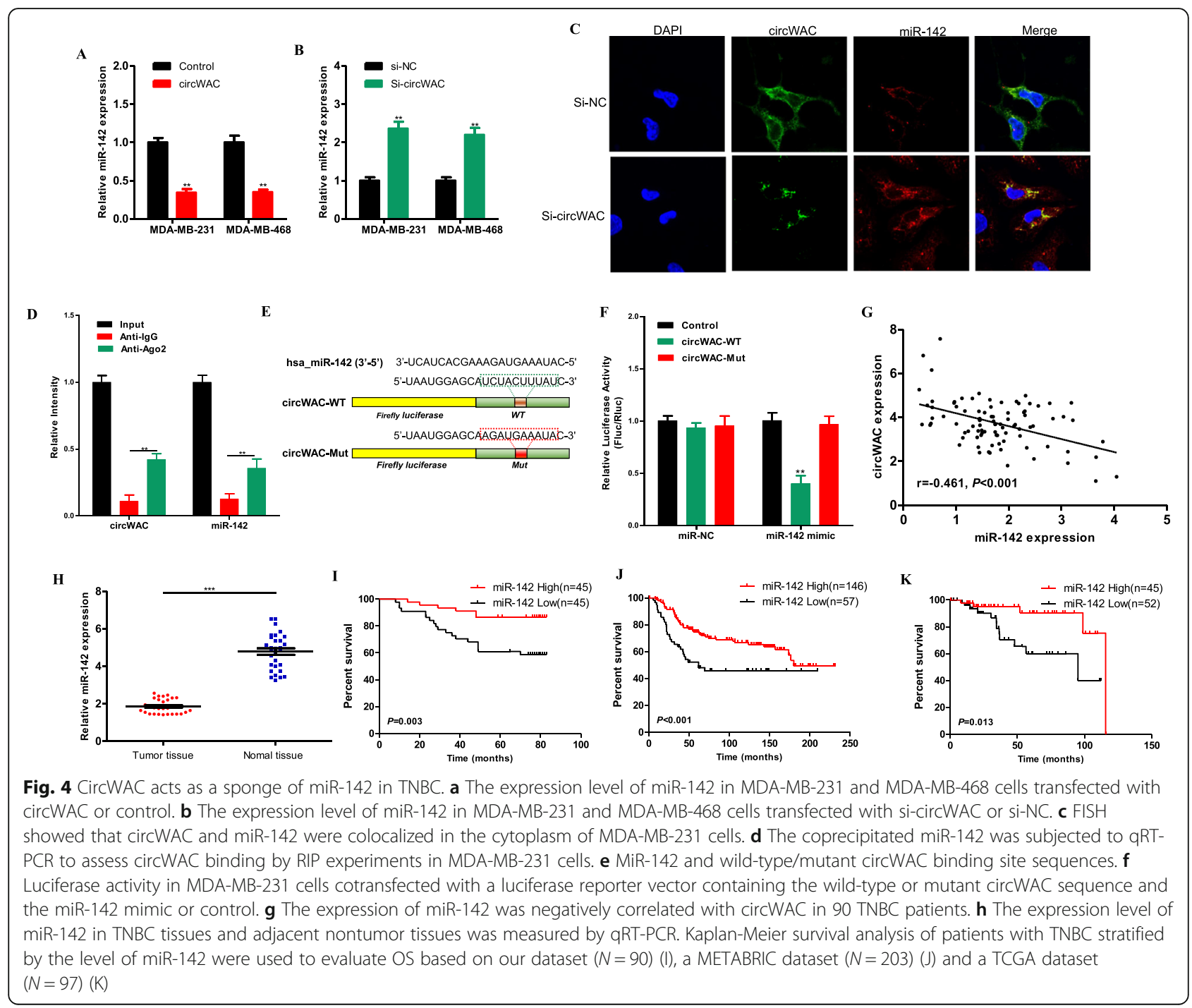


luciferase activity of the circWAC-WT vector but did not reduce the luciferase activity of the empty vector or the circWAC-MUT vector (Fig. 4f), confirming that circWAC can directly interact with miR-142. In addition, we performed a correlation analysis between circWAC and miR-142 based on 90 TNBC tissues. The expression of miR-142 was negatively correlated with circWAC in TNBC (Fig. 4g).

MiR-142 has been confirmed to play a role as a tumor suppressor in a variety of cancers $[11,12]$. The expression level of miR-142 was evaluated by qRT-PCR in 30 paired TNBC tumor tissues and adjacent tissue samples. The expression level of miR-142 in tumor tissues was significantly lower than that in adjacent nontumor tissues (Fig. 4h). Then, the expression level of miR-142 in 90 samples of TNBC was analyzed by qRT-PCR, and the survival outcomes of the corresponding patients were analyzed. The median expression level was used as the cutoff value, and it was found that the OS of TNBC patients with high miR-142 expression was significantly better than that of patients with low miR-142 expression (Fig. 4i). Subsequently, the data of TNBC patients in the METABRIC and TCGA databases were used for verification (the relevant data were downloaded from KaplanMeier Plotter, http://kmplot.com/analysis/), and the same results were obtained (Fig. 4j, k).

To determine whether circWAC regulates breast cancer chemosensitivity through miR-142, a gain-of-function assay was conducted. MDA-MB-231 and MDA-MB-468 cells were transfected to generate the following 4 groups: (1) control + miR-NC, (2) circWAC + miR-NC, (3) control + miR-142 mimic, and (4) circWAC + miR-142 mimic. qRT-PCR was then used to verify the expression of circWAC and miR-142 after transfection (Fig. 5a, b). The transfected cells were incubated with different concentrations of PTX $(0,5,10,15,20,25,30 \mu \mathrm{mol} / \mathrm{L})$ for 24 $\mathrm{h}$, and a CCK-8 assay was used to evaluate cell survival. The results showed that overexpression of circWAC reduced the PTX sensitivity of cells compared with that in the control group (Fig. 5c, d). In contrast, overexpression of miR-142 increased the sensitivity of the cells to PTX (Fig. 5c, d). However, in cells overexpressing miR-142, upregulation of circWAC did not reverse the chemosensitivity induced by miR-142 (Fig. 5c, d). Subsequent colony formation assays and apoptosis assays conducted with a PTX concentration of $20 \mu \mathrm{mol} / \mathrm{L}$ confirmed these results (Fig. 5e, f). Therefore, it is believed that miR-142 can block the induction of chemotherapeutic resistance by circWAC, indicating that the role of circWAC in PTX resistance in TNBC cells must depend on miR-142.

\section{WWP1 was the direct target gene of miR-142}

TargetScan (http://www.targetscan.org/), miRDB (http:// www.mirdb.org/) and miRNAMAP (http://mirnamap. mbc.nctu.edu.tw/) were used to predict the potential target genes of miR-142. The top 150 target genes predicted by the three databases were analyzed, and the 4 most promising target genes of miR-142 predicted by all three databases were identified: WWP1, DNAJC7, ATXN7L2 and NFE2L2 (Fig. 6a). These genes were evaluated with a RIP assay in MDA-MB-231 cells to determine whether their mRNA can bind to miR-142. The results showed that compared with that in the IgG control group, the mRNA level of WWP1 in the Ago2 antibody group was significantly increased (Fig. 6b). Subsequently, the miR-142 mimic or miR-142 inhibitor was transfected into MDA-MB-231 and MDA-MB-468 cells, and Western blotting was used to detect the changes in WWP1 protein expression levels. It was found that overexpression of miR-142 decreased the protein expression level of WWP1, while downregulation of miR-142 increased the protein expression level of WWP1 (Fig. 6c). Then, plasmids containing the wildtype sequence (WWP1-WT) or the mutant binding site sequence (WWP1-MUT) were constructed (Fig. 6d) and cotransfected with the miR-142 mimic or miR-NC into MDA-MB-231 cells for a dual luciferase reporter assay. The results showed that overexpression of miR-142 significantly reduced the luciferase activity of the vector containing WWP1-WT but did not reduce the luciferase activity of the empty vector or the vector containing WWP1-MUT (Fig. 6e), confirming that WWP1 is the direct target gene of miR-142.

WWP1 has been confirmed to act as an oncogene in a variety of cancers [13-18], including breast cancer [15-18]. High expression of WWP1 regulates PTEN polyubiquitination, thereby promoting activation of the PI3K/AKT signaling pathway [14]. The protein expression level of WWP1 in 90 TNBC samples was examined by IHC, and it was found that WWP1 expression in TNBC tissues was higher than that in adjacent normal tissues (Fig. 6f). Survival analysis was subsequently performed, and the median expression level was used as the cutoff value. The results indicated that the OS of TNBC patients with high WWP1 expression was significantly poorer than that of patients with low WWP1 expression (Fig. 6g).

To determine whether miR-142 regulates breast cancer chemosensitivity through WWP1, a gain-of-function assay was conducted. MDA-MB-231 and MDA-MB-468 cells were transfected to generate the following 4 groups: (1) control + miR-NC, (2) control + miR-142 mimic, (3) WWP1 + miR-NC, and (4) WWP1 + miR-142 mimic. Western blot analysis was used to verify the levels of WWP1, PTEN, AKT and pS473-AKT after transfection. The results showed that overexpression of WWP1 induced the phosphorylation of AKT at S473 (Fig. 7a), indicating that WWP1 overexpression can activate the PI3K/AKT signaling pathway, while overexpression of 


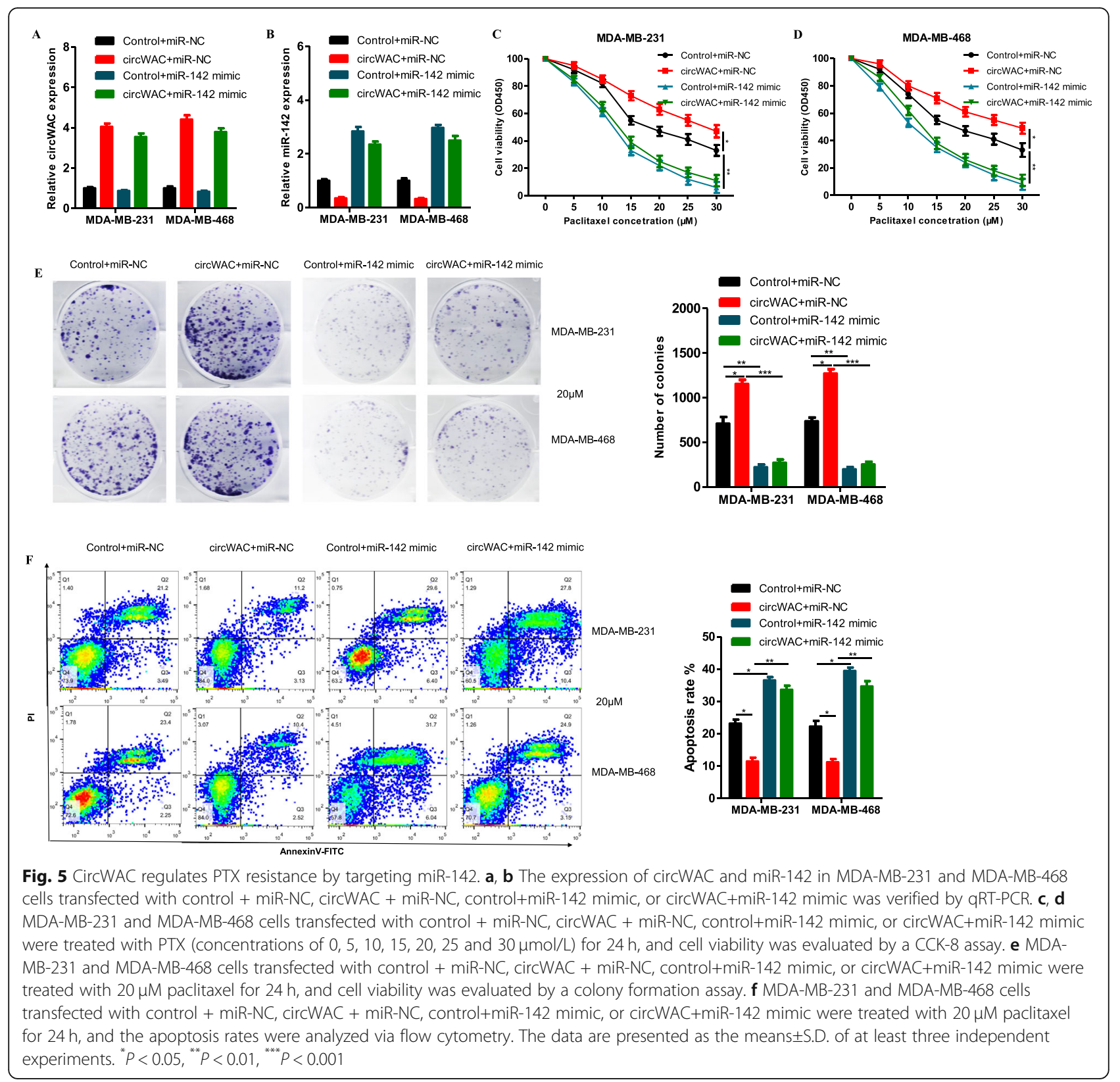

miR-142 inhibited the expression of WWP1 and the phosphorylation of AKT at S473 (Fig. 7a), indicating that miR-142 overexpression can inhibit the PI3K/AKT signaling pathway. The transfected cells were incubated with different concentrations of PTX $(0,5,10,15,20$, $25,30 \mu \mathrm{mol} / \mathrm{L}$ ) for $24 \mathrm{~h}$, and a CCK- 8 assay was used to evaluate cell survival. The results showed that overexpression of WWP1 could reduce the PTX sensitivity compared with that in the control group. In WWP1overexpressing cells, upregulation of miR-142 could not reverse the chemotherapeutic resistance induced by WWP1 (Fig. 7b, c). Subsequent colony formation assays and apoptosis assays conducted with a PTX concentration of $20 \mu \mathrm{mol} / \mathrm{L}$ confirmed these results (Fig. $7 \mathrm{~d}, \mathrm{e})$. Therefore, it is believed that WWP1 can block the induction of chemosensitivity by miR-142, indicating that the role of miR-142 in PTX resistance in TNBC cells must depend on WWP1. In summary, circWAC is an oncogene acting as a ceRNA to indirectly upregulate the expression of the target gene of miR-142, WWP1, by competitively binding miR-142 and activating the PI3K/ AKT pathway (Fig. 8).

\section{Inhibition of circWAC increases sensitivity to PTX in vivo} To verify whether inhibiting circWAC in TNBC cells can increase their sensitivity to PTX, the following 


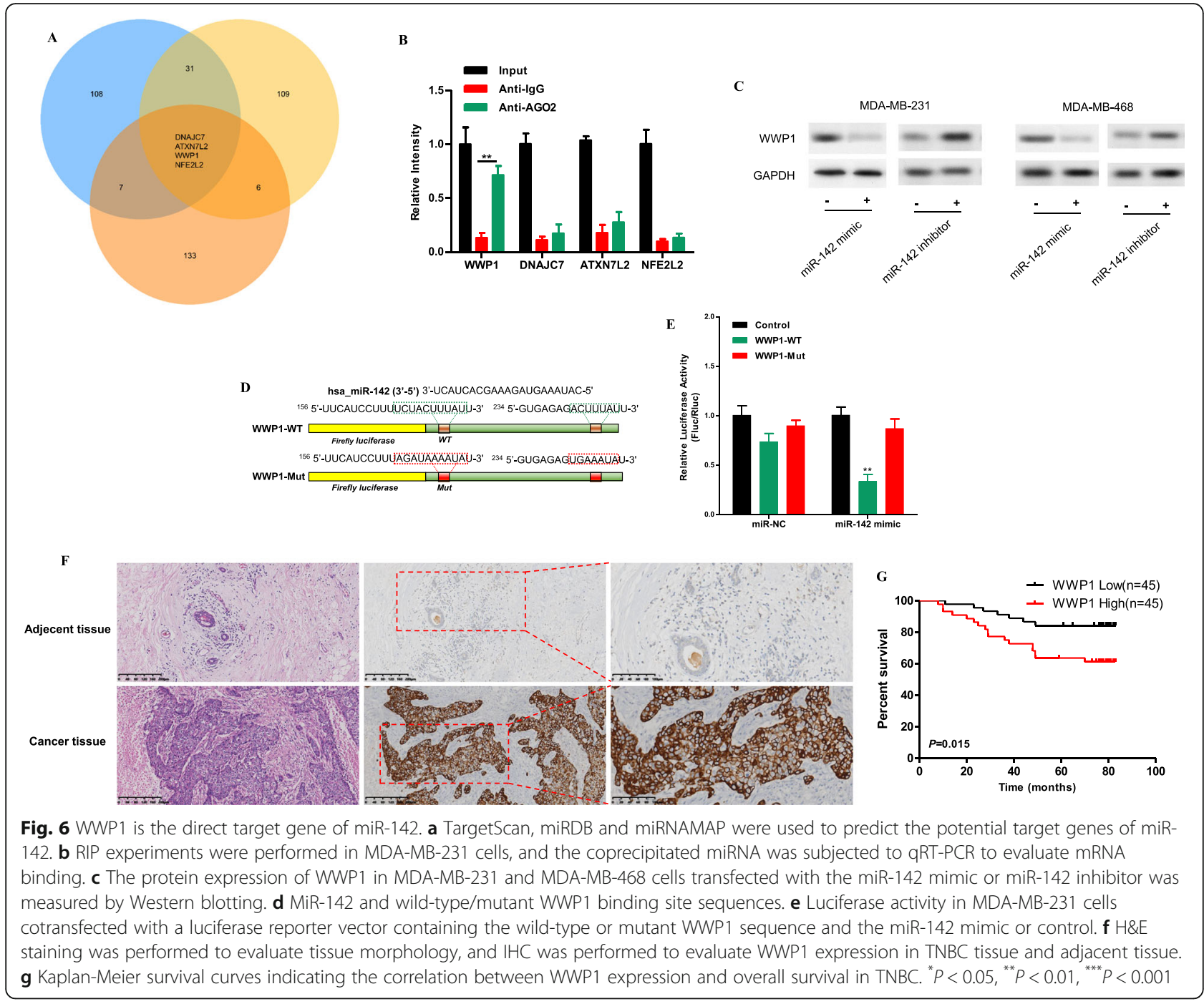

animal experiment was conducted. Mice were divided into the following four groups (see the Methods section for details): sh-NC + control, sh-circWAC + control, shNC + PTX, and sh-circWAC + PTX. The tumors in shcircWAC + PTX group mice were significantly smaller than those in sh-NC + PTX group mice, suggesting that inhibition of circWAC significantly increased chemosensitivity to PTX in the mice (Fig. 9a, b). Then, the expression of circWAC, miR-142 and WWP1 was evaluated in tumor tissues by qRT-PCR. It was found that circWAC and WWP1 were downregulated while miR-142 was highly expressed in the sh-circWAC and sh-circWAC + PTX groups, indirectly proving that circWAC competitively binds to miR-142 to upregulate the expression of WWP1 (Fig. 9c). Then, IHC and Western blotting were used to detect the levels of WWP1 and pS473-AKT in tumor tissues, and it was found that WWP1 and pS473AKT were expressed at low levels in the sh-circWAC and sh-circWAC +PTX groups (Fig. 9d, e), proving that decreased expression of WWP1 causes inactivation of the PI3K/AKT signaling pathway and leads to an increase in the sensitivity of breast cancer cells to chemotherapy.

\section{Discussion}

In the treatment of TNBC, PTX-based regimens have proven to be an important chemotherapeutic approach. Mechanistically, PTX treatment acts by inducing mitotic arrest by stabilizing spindle-shaped microtubules, which are responsible for the separation of replicated chromosomes into the daughter cell. However, the application of PTX-based chemotherapy in TNBC is limited by the emergence of PTX resistance. As noncoding molecules, circRNAs have been shown to play important roles in the occurrence and development of tumors [19]. Previous reports have identified a number of abnormally expressed circRNAs in TNBC that are upregulated in TNBC tissues and function as oncogenes. For example, 


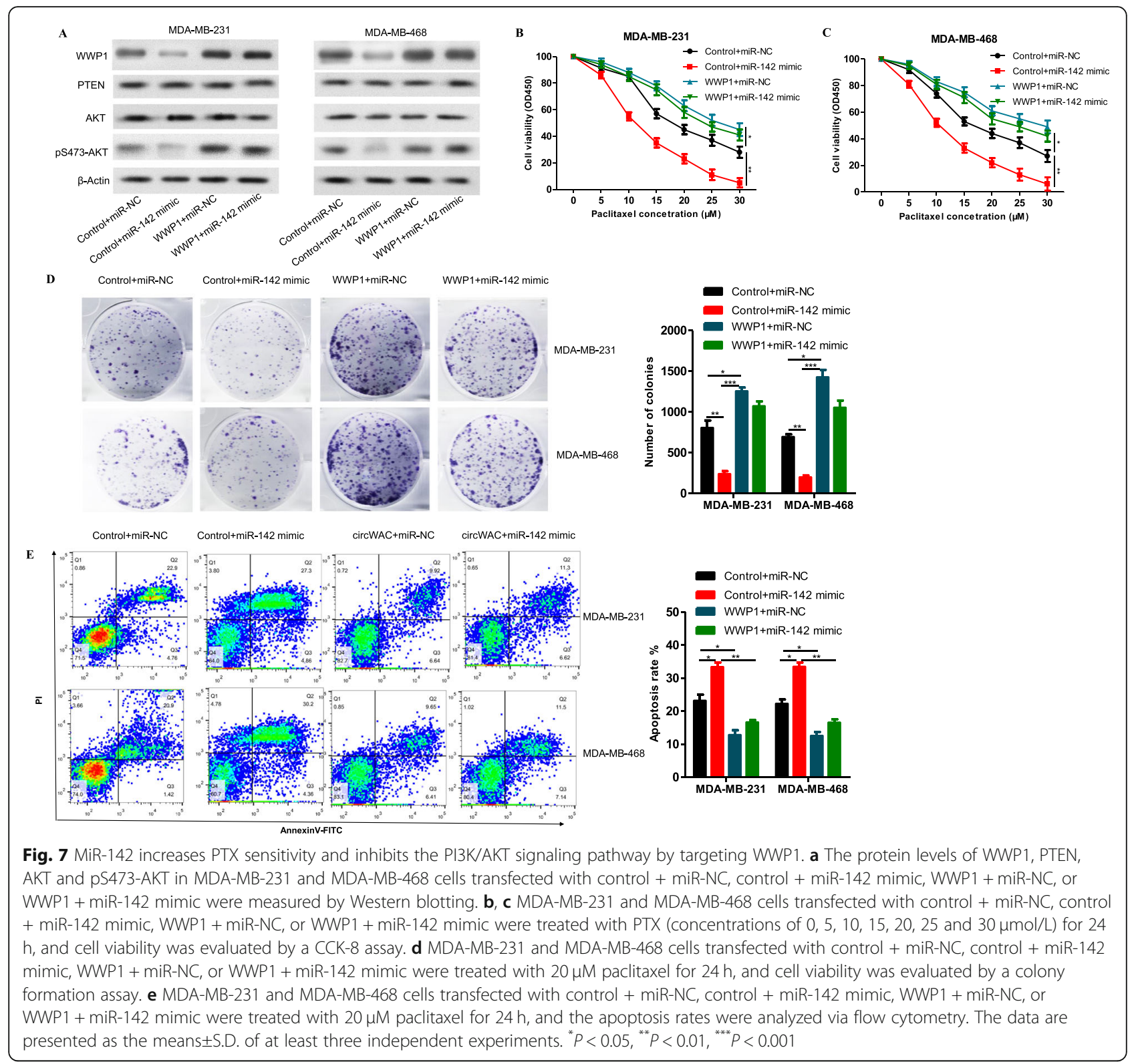

circGFRA1 can promote tumor proliferation and inhibit apoptosis by binding to miR-34a and upregulating the expression of GFRA1 in TNBC [20]. CircEPSTI1 can increase BCL11A expression in TNBC by sponging miR4753 and miR-6809, thus promoting cell proliferation and inhibiting apoptosis [21]. Zeng et al. found that circANKS1B is significantly upregulated in TNBC and that upregulation of circANKS1B expression is closely related to lymph node metastasis [22]. In vitro experiments showed that circANKS1B upregulates the expression of USF1 by sponging miR-148a-3p and miR-152-3p, which leads to upregulation of TGF- $\beta 1 / \mathrm{Smad}$ signaling and promotes EMT [22]. In addition, it was found that circAGFG1, circKIF4A, circPLK1 and circRAD18 can promote the malignant progression of TNBC [23-26].
Several studies have shown that circRNAs also play a role in suppressing TNBC. CircITCH is significantly downregulated in TNBC tissue, and downregulated circITCH expression is closely related to the poor prognosis of patients [27]. Overexpression of circITCH can upregulate ITCH1 by sponging miR-214 and miR-17, thereby inactivating $\mathrm{Wnt} / \beta$-catenin signaling [27]. Xu et al. found that upregulating the expression of circTADA2A-E6 inhibits cell proliferation, migration and colony formation via regulation of the miR-203a-3p/ SOCS3 signaling pathway [10]. CircFBXW7 inhibits the malignant progression of TNBC by upregulating miR197-3p and encoding the tumor suppressor FBXW7185aa [28]. These circRNAs involved in TNBC, which mostly affect the proliferation, invasion and metastasis 


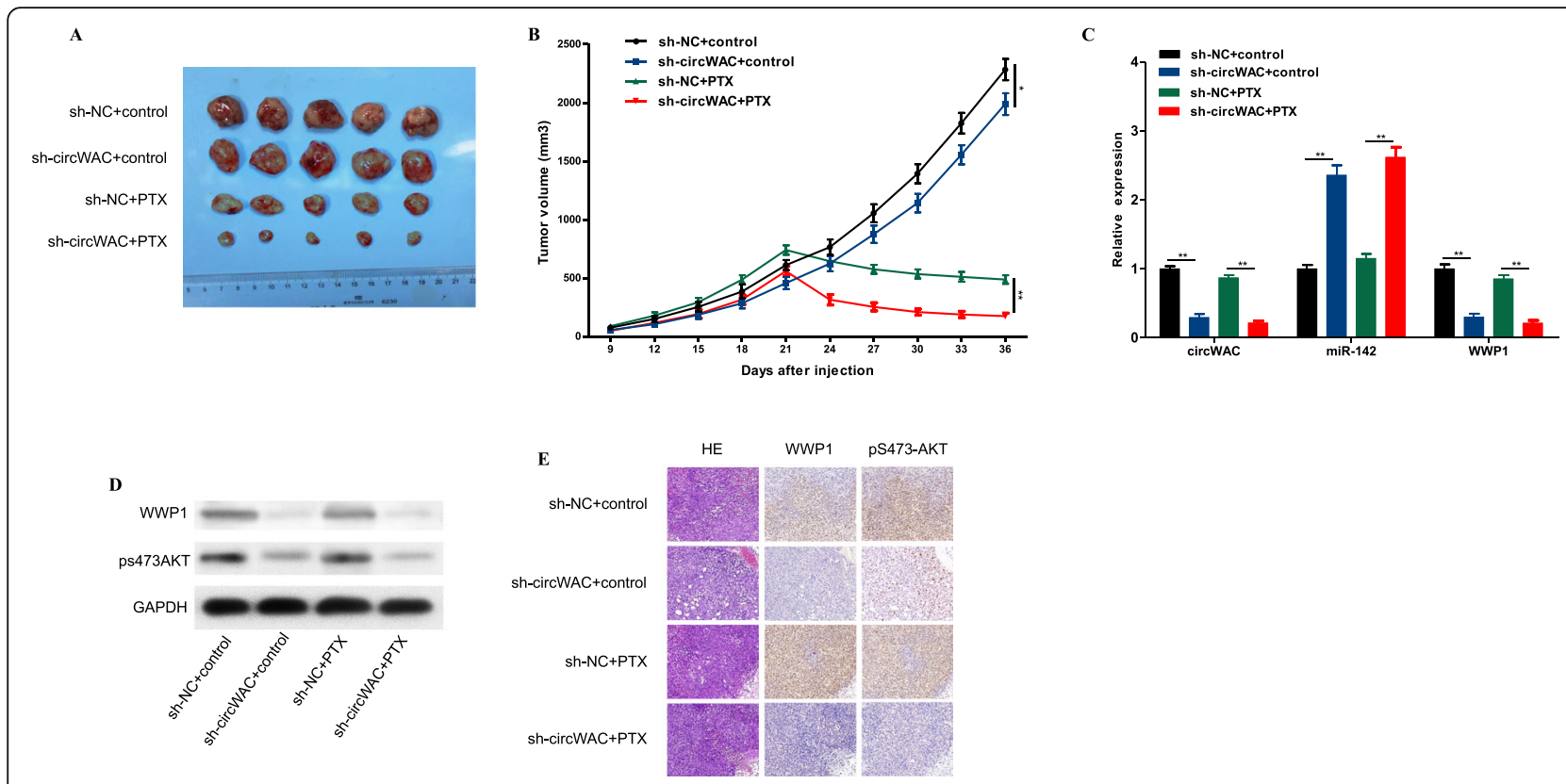

Fig. 8 A summary map to illustrate the interaction relation among these molecules and signal pathways to drug resistance in TNBC

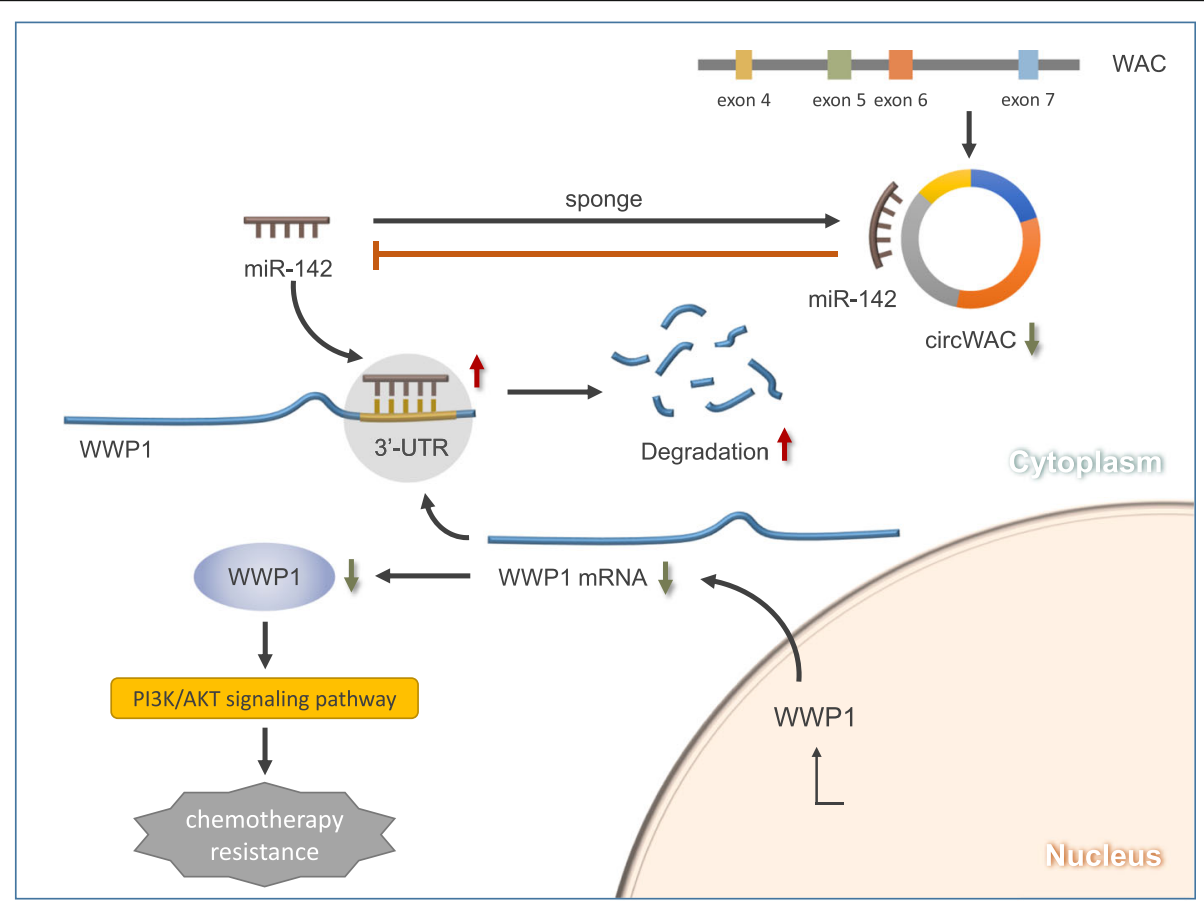

Fig. 9 Inhibition of circWAC can increase chemosensitivity to PTX in vivo. a Image of representative resected tumors from four groups of xenografted nude mice on the 36th day. b Tumor volumes were monitored during the 36-d time course. $\mathbf{c}$ The expression levels of circWAC, miR-142 and WWP1 in tumor tissues were determined by qRT-PCR. $\mathbf{d}$ The levels of WWP1 and PS473-AKT in tumor tissues were detected by Western blotting. e H\&E staining was performed to evaluate tissue morphology, and IHC was performed to visualize WWP1 and PS473-AKT. ${ }^{*} P<$ $0.05,{ }^{* * *} P<0.01,{ }^{* * *} P<0.001$ 
of tumor cells through the ceRNA mechanism, are closely related to the clinicopathological factors of TNBC patients and have potential as prognostic indexes of TNBC. In this study, it was found that circWAC was highly expressed in TNBC and was associated with poor TNBC patient prognosis. Then, the results of experiments in cells and animal models verified that downregulation of circWAC increases the sensitivity of TNBC cells to PTX.

Next, the molecular mechanism of circWAC in TNBC chemotherapeutic resistance was explored and verified. First, candidate miRNAs with sequences complementary to circWAC were screened through bioinformatic approaches, and it was found that the expression of miR-142 was also negatively regulated by circWAC in TNBC cells. RNA FISH showed that circWAC and miR-142 colocalized in the cytoplasm of MDA-MB-231 cells. Furthermore, RIP and dual luciferase reporter assays proved a direct interaction between circWAC and miR-142. In the gain-of-function assay, miR-142 blocked the induction of chemotherapeutic resistance in TNBC cells by circWAC. In addition, the OS of TNBC patients with high expression of miR-142 was significantly better than that of patients with low expression of miR-142, and these results were verified in the METABRIC and TCGA databases. MiR-142 has been confirmed to play a role as a tumor suppressor in a variety of cancers $[11,12$, $29,30]$. Low expression of miR-142 is closely related to the high recurrence and poor prognosis of gastric cancer [12]. Pancreatic cancer patients with high expression of miR-142 have longer OS times than those with low expression of this miRNA [29]. Tsang et al. found that miR-142 is expressed at significantly lower levels in HCC tissue than in non-HCC tissue and that high expression of miR-142 can inhibit the proliferation and migration of HCC cells [30]. Jia et al. found that miR-142 inhibitors can enhance the ability of lung adenocarcinoma cells to undergo EMT [11]. Finally, bioinformatic analyses and cell experiments were performed to identify target genes regulated by miR-142, and it was proven that both WWP1 expression and PI3K/AKT pathway activity were regulated by circWAC and miR-142. Gain-of-function assays confirmed that WWP1 can block the chemosensitivity of TNBC cells through miR-142. In addition, it was confirmed that WWP1 was highly expressed in TNBC and that the prognosis of patients with high WWP1 expression was poor. Previously, WWP1 was shown to be overexpressed or amplified in breast cancer, indicating that it acts as an oncogene [15-18]. Recently, WWP1 has been proven to act as a switch to reactivate PTEN. Downregulation of WWP1 leads to inactivation of the PI3K/AKT signaling pathway $[13,14]$.

\section{Conclusion}

In summary, circWAC/miR-142/WWP1 can form a ceRNA network to regulate PI3K/AKT signaling activity in TNBC cells and affect their chemosensitivity. Based on this mechanism, it is believed that the circWAC/ miR-142/WWP1 axis has great potential as a new biomarker and new therapeutic target for TNBC.

\section{Supplementary Information}

The online version contains supplementary material available at https://doi. org/10.1186/s12943-021-01332-8.

\begin{abstract}
Additional file 1: Supplementary Table 1. Primers for qRT-PCR. Supplementary Table 2. RNA probes for FISH.

Additional file 2: Supplementary Figure 1. A. Cell invasion ability of MDA-MB-231 cells transfected with Si-NC or Si-circWAC was evaluated by transwell invasion assays. B. Wound healing assays of MDA-MB-231 cells transfected with Si-NC or Si-circWAC were performed to evaluate cell migration ability. Supplementary Figure 2. A-D. Cells were treated with cisplatin (concentrations of 0, 2.5, 5, 7.5, 10 and $12.5 \mu \mathrm{mol} / \mathrm{L}$ ) for $24 \mathrm{~h}$, and cell viability was evaluated by a CCK-8 assay. E-H. Cells were treated with cisplatin $(0$ and $10 \mu \mathrm{M})$ for $24 \mathrm{~h}$, and cell viability was evaluated by a colony formation assay. I-J. Cells were treated with cisplatin ( 0 and $10 \mu \mathrm{M})$ for $24 \mathrm{~h}$, and the apoptosis rate was analyzed via flow cytometry. The data are presented as the means \pm S.D. of at least three independent experiments. ${ }^{*} P<0.05,{ }^{*} P<0.01,{ }^{* *} P<0.001$
\end{abstract}

\section{Abbreviations \\ Ago: Argonaute 2; CCK-8: Cell Counting Kit-8; cDNA: Complementary DNA; ceRNAs: Competing endogenous RNAs; circRNAs: Circular RNAs; RNA FISH: RNA fluorescence in situ hybridization; gDNA: Genomic DNA; H\&E: Hematoxylin and eosin; IHC: Immunohistochemical staining; OS: Overall survival; PTX: Paclitaxel; qRT-PCR: Quantitative real-time PCR; RIP: RNA immunoprecipitation; siRNAs: Short interfering RNAs; TNBC: Triple-negative breast cancer; WWP1: WW domain-containing ubiquitin E3 ligase 1}

\section{Acknowledgments}

Not applicable.

\begin{abstract}
Authors' contributions
L.W., Y.H.Z., Y.C. and L.F.Z. conceived and designed the study and helped draft the manuscript. L.L.L. and T.T.D. performed the data collection. L.W., Y.H.Z., L.J. and A.L. performed the experimental work. T.T.D. performed the statistical analysis. All authors read and critically revised the manuscript for intellectual content and approved the final manuscript.
\end{abstract}

\section{Funding}

This work was financially supported by the Suzhou Health Science and Technology Project (Gwzx201703).

\section{Availability of data and materials}

The circular RNA sequencing datasets were obtained from the Gene Expression Omnibus database (http://www.ncbi.nlm.nih.gov/geo/) under the series accession number GSE101124. MiR-142-related data from the METABRIC and TCGA databases were downloaded from Kaplan-Meier Plotter (http://kmplot.com/analysis/). All data and materials supporting the conclusions of this study have been included within the article and the supplemental data.

\section{Ethics approval and consent to participate}

All animal experiments were approved by the ethical review committee of the First Affiliated Hospital of Soochow University. The study protocol was performed in accordance with the guidelines outlined in the Declaration of Helsinki. The Ethics Committee of the First Affiliated Hospital of Soochow University approved the study, and all participants signed informed consent statements. 


\section{Consent for publication}

Not applicable.

\section{Competing interests}

The authors declare that they have no competing interests.

\section{Author details}

'Department of General Surgery, The First Affiliated Hospital of Soochow University, 188 Shizi Street, Suzhou 215000, China. ${ }^{2}$ Insitute of Blood Transfusion, Suzhou Blood Center, Suzhou 215000, China.

Received: 14 August 2020 Accepted: 9 February 2021

Published online: 01 March 2021

\section{References}

1. Bray F, Ferlay J, Soerjomataram I, Siegel RL, Torre LA, Jemal A. Global cancer statistics 2018: GLOBOCAN estimates of incidence and mortality worldwide for 36 cancers in 185 countries. CA Cancer J Clin. 2018;68:394-424.

2. Chen W, Sun K, Zheng R, Zeng H, Zhang S, Xia C, Yang Z, Li H, Zou X, He J. Cancer incidence and mortality in China, 2014. Chin J Cancer Res. 2018;30:1-12.

3. Fan L, Strasser-Weippl K, Li JJ, St Louis J, Finkelstein DM, Yu KD, Chen WQ, Shao ZM, Goss PE. Breast cancer in China. Lancet Oncol. 2014;15:e279-89.

4. Chen W, Zheng R, Baade PD, Zhang S, Zeng H, Bray F, Jemal A, Yu XQ, He J. Cancer statistics in China, 2015. CA Cancer J Clin. 2016;66:115-32.

5. Yoon HJ, Jun CD, Kim JM, Rim GN, Kim HM, Chung HT. Phorbol ester synergistically increases interferon-gamma-induced nitric oxide synthesis in murine microglial cells. Neuroimmunomodulation. 1994;1:377-82.

6. Bianchini G, Balko JM, Mayer IA, Sanders ME, Gianni L. Triple-negative breast cancer: challenges and opportunities of a heterogeneous disease. Nat Rev Clin Oncol. 2016;13:674-90.

7. Vicens Q, Westhof E. Biogenesis of circular RNAs. Cell. 2014;159:13-4.

8. Hansen TB, Kjems J, Damgaard CK. Circular RNA and miR-7 in cancer. Cancer Res. 2013;73:5609-12.

9. Misir S, Hepokur C, Aliyazicioglu Y, Enguita FJ. Circular RNAs serve as miRNA sponges in breast cancer. Breast Cancer. 2020;27(6):1048-57.

10. Xu JZ, Shao CC, Wang XJ, Zhao X, Chen JQ, Ouyang YX, Feng J, Zhang F, Huang WH, Ying Q, et al. circTADA2As suppress breast cancer progression and metastasis via targeting miR-203a-3p/SOCS3 axis. Cell Death Dis. 2019;10:175.

11. Jia Y, Duan Y, Liu T, Wang X, Lv W, Wang M, Wang J, Liu L. LncRNA TTN-AS1 promotes migration, invasion, and epithelial mesenchymal transition of lung adenocarcinoma via sponging miR-142-5p to regulate CDK5. Cell Death Dis. 2019;10:573.

12. Zhang X, Yan Z, Zhang J, Gong L, Li W, Cui J, Liu Y, Gao Z, Li J, Shen L, Lu Y. Combination of hsa-miR-375 and hsa-miR-142-5p as a predictor for recurrence risk in gastric cancer patients following surgical resection. Ann Oncol. 2011;22:2257-66.

13. Lee YR, Yehia L, Kishikawa T, Ni Y, Leach B, Zhang J, Panch N, Liu J, Wei W, Eng C, Pandolfi PP. WWP1 gain-of-function inactivation of PTEN in Cancer predisposition. N Engl J Med. 2020;382:2103-16.

14. Lee YR, Chen M, Lee JD, Zhang J, Lin SY, Fu TM, Chen H, Ishikawa T, Chiang SY, Katon J, et al. Reactivation of PTEN tumor suppressor for cancer treatment through inhibition of a MYC-WWP1 inhibitory pathway. Science. 2019;364:eaau0159.

15. Chen C, Zhou Z, Sheehan CE, Slodkowska E, Sheehan CB, Boguniewicz A, Ross JS. Overexpression of WWP1 is associated with the estrogen receptor and insulin-like growth factor receptor 1 in breast carcinoma. Int J Cancer. 2009;124:2829-36.

16. Li Y, Zhou Z, Chen C. WW domain-containing E3 ubiquitin protein ligase 1 targets p63 transcription factor for ubiquitin-mediated proteasomal degradation and regulates apoptosis. Cell Death Differ. 2008;15:1941-51.

17. Chen C, Zhou Z, Liu R, Li Y, Azmi PB, Seth AK. The WW domain containing E3 ubiquitin protein ligase 1 upregulates ErbB2 and EGFR through RING finger protein 11. Oncogene. 2008;27:6845-55.

18. Chen C, Zhou Z, Ross JS, Zhou W, Dong JT. The amplified WWP1 gene is a potential molecular target in breast cancer. Int J Cancer. 2007;121:80-7.

19. Li J, Sun D, Pu W, Wang J, Peng Y. Circular RNAs in Cancer: biogenesis, function, and clinical significance. Trends Cancer. 2020;6:319-36.

20. He R, Liu P, Xie X, Zhou Y, Liao Q, Xiong W, Li X, Li G, Zeng Z, Tang H. circGFRA1 and GFRA1 act as ceRNAs in triple negative breast cancer by regulating miR-34a. J Exp Clin Cancer Res. 2017;36:145.
21. Chen B, Wei W, Huang X, Xie X, Kong Y, Dai D, Yang L, Wang J, Tang H. circEPSTI1 as a prognostic marker and mediator of triple-negative breast Cancer progression. Theranostics. 2018;8:4003-15.

22. Zeng K, He B, Yang BB, Xu T, Chen X, Xu M, Liu X, Sun H, Pan Y, Wang S. The pro-metastasis effect of circANKS1B in breast cancer. Mol Cancer. 2018;17:160.

23. Yang $R$, Xing $L$, Zheng $X$, Sun $Y$, Wang $X$, Chen J. The circRNA circAGFG1 acts as a sponge of miR-195-5p to promote triple-negative breast cancer progression through regulating CCNE1 expression. Mol Cancer. 2019;18:4.

24. Tang H, Huang X, Wang J, Yang L, Kong Y, Gao G, Zhang L, Chen ZS, Xie X. circKIF4A acts as a prognostic factor and mediator to regulate the progression of triple-negative breast cancer. Mol Cancer. 2019;18:23.

25. Kong Y, Yang L, Wei W, Lyu N, Zou Y, Gao G, Ou X, Xie X, Tang H. CircPLK1 sponges miR-296-5p to facilitate triple-negative breast cancer progression. Epigenomics. 2019;11:1163-76.

26. Zou Y, Zheng S, Xiao W, Xie X, Yang A, Gao G, Xiong Z, Xue Z, Tang H. circRAD18 sponges miR-208a/3164 to promote triple-negative breast cancer progression through regulating IGF1 and FGF2 expression. Carcinogenesis. 2019;40:1469-79.

27. Wang ST, Liu LB, Li XM, Wang YF, Xie PJ, Li Q, Wang R, Wei Q, Kang YH, Meng $\mathrm{R}$, Feng $\mathrm{XH}$. Circ-ITCH regulates triple-negative breast cancer progression through the Wnt/beta-catenin pathway. Neoplasma. 2019;66:232-9.

28. Ye F, Gao G, Zou Y, Zheng S, Zhang L, Ou X, Xie X, Tang H. circFBXW7 inhibits malignant progression by sponging miR-197-3p and encoding a 185-aa protein in triple-negative breast Cancer. Mol Ther Nucleic Acids. 2019;18:88-98.

29. Ohuchida K, Mizumoto K, Kayashima T, Fujita H, Moriyama T, Ohtsuka T, Ueda J, Nagai E, Hashizume M, Tanaka M. MicroRNA expression as a predictive marker for gemcitabine response after surgical resection of pancreatic cancer. Ann Surg Oncol. 2011;18:2381-7.

30. Tsang FH, Au SL, Wei L, Fan DN, Lee JM, Wong CC, Ng IO, Wong CM. MicroRNA-142-3p and microRNA-142-5p are downregulated in hepatocellular carcinoma and exhibit synergistic effects on cell motility. Front Med. 2015;9:331-43.

\section{Publisher's Note}

Springer Nature remains neutral with regard to jurisdictional claims in published maps and institutional affiliations.

Ready to submit your research? Choose BMC and benefit from:

- fast, convenient online submission

- thorough peer review by experienced researchers in your field

- rapid publication on acceptance

- support for research data, including large and complex data types

- gold Open Access which fosters wider collaboration and increased citations

- maximum visibility for your research: over $100 \mathrm{M}$ website views per year

At BMC, research is always in progress.

Learn more biomedcentral.com/submissions 